\title{
Smoking cessation in China: findings from the 1996 national prevalence survey
}

\author{
Gonghuan Yang, Jiemin Ma, Aiping Chen, Yifang Zhang, Jonathan M Samet, \\ Carl E Taylor, Karen Becker
}

\begin{abstract}
Objectives-To describe patterns of smoking and smoking cessation in China within the context of the stages of change model, using data from the 1996 national prevalence survey.

Design-A cross sectional survey was carried out using the 145 preselected disease surveillance points, which provide a representative sample for the entire country. A standardised questionnaire on smoking was interviewer administered.
\end{abstract} Setting-The country of China. Subjects-122 220 people aged 15-69 years.

Maintenance measures-Smoking cessation patterns, as defined by smoking status (current or former) and stage of change (precontemplation, contemplation, and action).

Results-The sample included 45995 ever smokers of whom 4336 had quit. About $72 \%$ of current smokers reported not intending to give up their smoking behaviour, and about $16 \%$ of current smokers said they intended to do so, but have not taken any action. Of all ever smokers, the percentage of former smokers was $9.5 \%$, and $12 \%$ of current smokers had quit at least once, but relapsed by the time of the survey. The patterns were similar in men and women with regard to the stated intent to quit. Among males, the percentage of former smokers increased with age but the percentage intending to quit was constant at about $15 \%$ across age strata. The most common reason for quitting was illness. Participants with a university education were more likely to have made an attempt to quit.

Conclusions-The percentage of smokers contemplating quitting was low in China in 1996. The study shows that smokers in China must be mobilised to contemplate quitting and then to take action.

(Tobacco Control 2001;10:170-174)

Keywords: China; cessation; smoking patterns

China is the world's largest producer and consumer of tobacco. ${ }^{1}$ Prevalence rates in the 1996 national prevalence survey for ever smokers were $66.9 \%$ for males and $4.2 \%$ for females, with an overall prevalence of $37.6 \%$ among China's population 15 years of age and above. ${ }^{2}$ The 1996 results translate to approximately 320 million ever smokers in China, 300 million men and 20 million women of the 860 million people aged 15 and over. ${ }^{3}$ Comparison of the
1996 results with the 1984 national prevalence survey shows a rise in smoking among younger males across the 12 years $^{3}$, while rates have not increased in women.

The effects of smoking on health in China are already evident. Recent large studies showed that smoking increases risk for many diseases and raises total mortality. ${ }^{45} \mathrm{At}$ present, the proportion of deaths in adult males attributed to tobacco smoking is $12 \%$, which is projected to rise to $33 \%$ by 2030 if smoking continues at present rates. ${ }^{67}$ These estimates indicate that in China one million people will die annually of diseases related to smoking in the year 2000, two million by around 2025, and three million by around 2050 if present smoking patterns persist.

Some of these deaths can be prevented through smoking cessation, an essential component of tobacco control within a comprehensive intervention strategy. There are many barriers to effective cessation, particularly the addictive nature of nicotine. ${ }^{8}$ Cessation of smoking is a complex, dynamic process, formulated as including the stages of precontemplation, contemplation, preparation, action, and maintenance. ${ }^{9}$ A characterisation of the distribution of the population across these stages is essential for planning tobacco cessation programmes, as different interventions are appropriate to the different stages. In this paper, we describe the characteristics of Chinese ever smokers, addressing cessation and intent to quit in the 1996 national prevalence survey.

\section{Methods}

The survey was conducted in a population of about 10 million, living in 145 selected disease surveillance points (DSPs) in the 30 provinces of China. The geographic locations in the DSP system were selected in 1989 by multi-stage random sampling with stratification at three levels (major geographic regions of China, urban or rural, and four categories derived from nine socioeconomic, demographic, and mortality indicators). The unit of sampling was the district, and probability of selection was determined by population size. The DSP sample has been documented to be a representative sample of the Chinese population. ${ }^{10}$

The sample for the 1996 national prevalence survey was selected from the DSP population. In each DSP, 1000 persons aged 15-69 years were selected by a three stage procedure $(20$ neighbourhoods per DSP, 50 households per neighbourhood, one person per household). The overall response rate was $94.9 \%$. After deleting some records with missing values, there were 122220 records for analysis. 
Table 1 Distributions of smokers by stage of quitting and age groups in 1996 survey in China

\begin{tabular}{|c|c|c|c|c|c|}
\hline & & & Intent to qu & & \\
\hline & (years) & $n$ & Not intend & Intend & Relapse \\
\hline Males & Total & 39551 & 28388 & 6585 & 4579 \\
\hline & & & $71.8(\%)$ & $16.6(\%)$ & $11.6(\%)$ \\
\hline & $15-19$ & 690 & 74.1 & 18.0 & 7.9 \\
\hline & $20-24$ & 2710 & 75.6 & 15.6 & 8.8 \\
\hline & $25-29$ & 5317 & 74.8 & 15.7 & 9.5 \\
\hline & $30-34$ & 6663 & 73.8 & 15.8 & 10.4 \\
\hline & $35-39$ & 5754 & 73.6 & 16.0 & 10.4 \\
\hline & $40-44$ & 5537 & 70.7 & 16.7 & 12.6 \\
\hline & $45-49$ & 3364 & 71.3 & 17.3 & 11.4 \\
\hline & $50-54$ & 2520 & 68.8 & 17.7 & 13.5 \\
\hline & $55-59$ & 2382 & 65.7 & 19.3 & 15.0 \\
\hline & $60-64$ & 2188 & 66.2 & 17.9 & 15.9 \\
\hline & $65+$ & 2436 & 67.4 & 17.1 & 15.5 \\
\hline Females & Total & 2095 & 1498 & 341 & 256 \\
\hline & & & $71.5(\%)$ & $16.3(\%)$ & $12.2(\%)$ \\
\hline & $15-19$ & 9 & 88.9 & 0.0 & 11.1 \\
\hline & $20-24$ & 31 & 64.4 & 25.8 & 9.7 \\
\hline & $25-29$ & 98 & 78.6 & 12.2 & 9.2 \\
\hline & $30-34$ & 177 & 66.7 & 20.9 & 12.4 \\
\hline & $35-39$ & 175 & 78.3 & 14.3 & 7.4 \\
\hline & $40-44$ & 190 & 71.6 & 15.8 & 12.6 \\
\hline & $45-49$ & 176 & 73.3 & 15.3 & 11.4 \\
\hline & $50-54$ & 189 & 77.3 & 12.7 & 10.1 \\
\hline & $55-59$ & 280 & 66.7 & 19.3 & 13.9 \\
\hline & $60-64$ & 361 & 70.6 & 15.8 & 13.6 \\
\hline & $65+$ & 408 & 69.6 & 16.4 & 13.9 \\
\hline
\end{tabular}

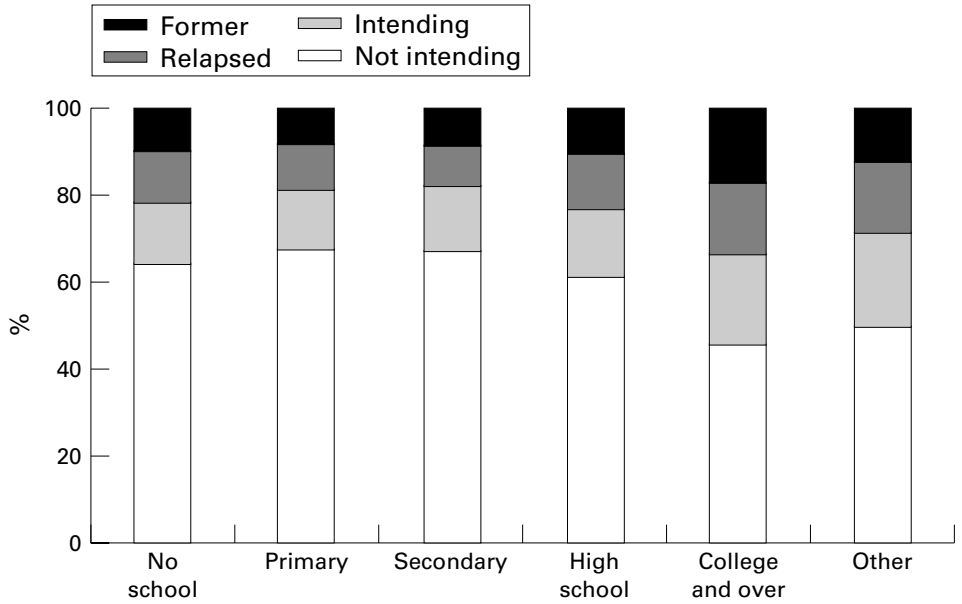

Figure 1 Percentage distribution by education of relapsed former smokers and former smokers at survey by duration of quitting.

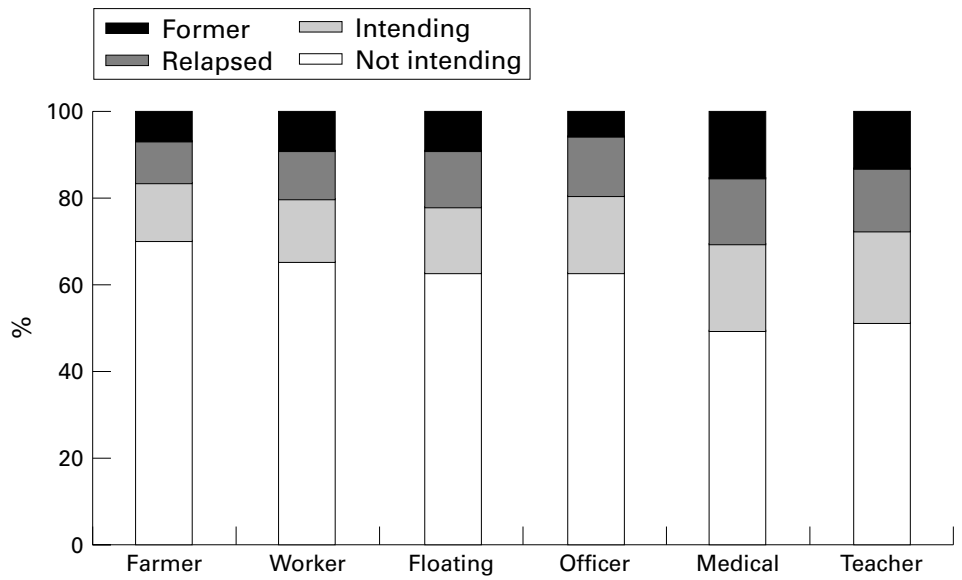

Figure 2 Percentage distribution by occupation of relapsed former smokers and former smokers at survey by duration of quitting.
The survey was accomplished by specially trained interviewers, who administered a 52 item questionnaire, which included information on demographics, smoking history, smoking related knowledge and attitudes, cessation, and health status. The indicators for ever smokers ${ }^{11}$ included persons who had ever smoked for at least six months during their lives; current smokers were smoking tobacco products at the time of the survey; and former smokers had smoked for at least six months but were not smoking at the time of the survey. To describe the profile of cessation, we asked current smokers if they had ever stopped and also if they intended to give up smoking. Using the responses to these two questions, we divided current smokers into three groups: never quit and not intending to quit; never quit and intending to quit; and relapsed quitters. We asked former smokers how long they had successfully quit (less than six months, less than one year, less than two years, two years and over, and don't know). ${ }^{12}$ We further classified quitters as taking action (quitting period of less than one month), maintenance (quitting period of more than one month and less than two years), and success (two years and longer). Relapsed quitters were not asked about intent to quit again.

We described differences in cessation profiles by age, educational level, occupational level, and urban or rural residence. Education level was classified as no school, primary school, secondary school, high school, and college and above. The survey participants were classified into 14 occupational groups. For this analysis, we considered farmers, workers, floating people who live in the urban areas but do not have fixed work and fixed residence cards, officers, medical workers, and teachers. These occupational groups comprised $76 \%$ of the total sample.

\section{Results}

The total sample of 122220 persons with complete data included 45995 ever smokers of whom 4336 were former smokers. Table 1 provides the numbers of current smokers by age group and the percentage distributions as those who had never contemplated quitting at the time of interview, those intending to quit, and relapsed quitters. Overall, about $71.8 \%$ of current smokers had no intention of stopping smoking; $16.6 \%$ of current smokers said they intended to do so but had not taken any action, and $11.6 \%$ had once quit but relapsed by the time of the survey. The patterns were similar in men and women with regard to cessation intent: in men, $71.8 \%$ not intending to quit, $16.6 \%$ intending to quit, and $11.6 \%$ relapsed. For women, the corresponding figures were $71.5 \%, 16.3 \%$, and $11.6 \%$, respectively. Stated intent to quit was associated with age group among males. In general the older male smokers had a higher proportion of quitters, and a lower proportion of persons not intending to quit. The profile was not associated with age group among females.

There were differences in the intent-to-quit profile across groups with different educational levels and occupations (figs 1 and 2). With 


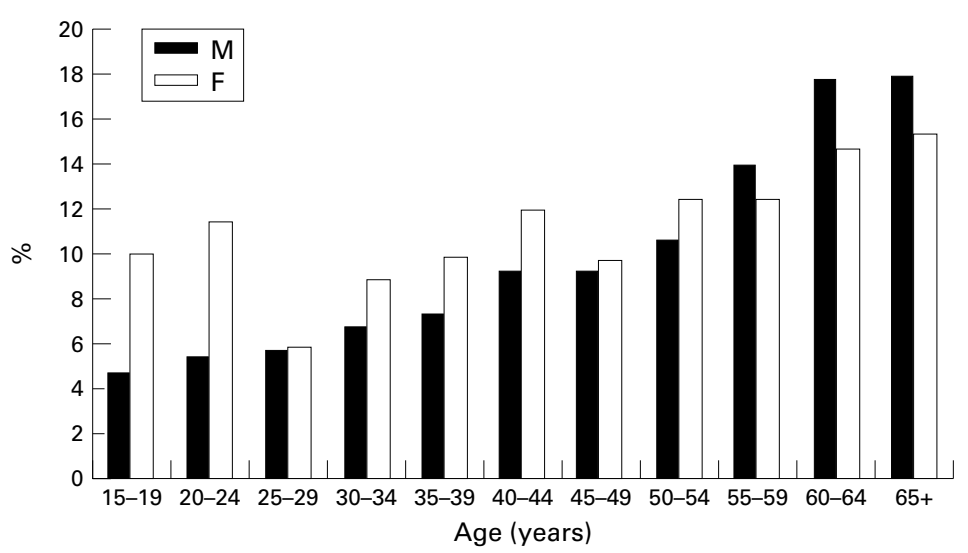

Figure 3 National projections for numbers of smokers by smoking status, intent to quit, and duration of quitting, based on 1996 national prevalence survey. M, males, F, females.

Table 2 Reasons for quitting smoking by age group in the 1996 survey on smoking in China

\begin{tabular}{llllrll}
\hline $\begin{array}{l}\text { Age } \\
\text { (years) }\end{array}$ & $\begin{array}{l}\text { Illness } \\
(\%)\end{array}$ & $\begin{array}{l}\text { Prevent illness } \\
(\%)\end{array}$ & $\begin{array}{l}\text { Family against } \\
(\%)\end{array}$ & Cost (\%) & $\begin{array}{l}\text { Education } \\
(\%)\end{array}$ & $\begin{array}{l}\text { Environmental } \\
(\%)\end{array}$ \\
\hline $15-19$ & 25.0 & 25.0 & 12.5 & 0.00 & 12.5 & 0.0 \\
$20-24$ & 17.7 & 26.5 & 14.7 & 14.71 & 11.8 & 5.9 \\
$25-29$ & 30.9 & 36.4 & 27.3 & 14.55 & 14.6 & 7.3 \\
$30-34$ & 26.9 & 41.2 & 16.8 & 16.81 & 13.5 & 3.4 \\
$35-39$ & 34.9 & 32.2 & 23.0 & 11.18 & 9.9 & 2.6 \\
$40-44$ & 31.6 & 40.8 & 18.4 & 12.64 & 14.4 & 3.5 \\
$45-49$ & 43.0 & 34.1 & 15.6 & 11.11 & 5.9 & 5.9 \\
$50-54$ & 46.5 & 28.4 & 16.5 & 17.32 & 13.4 & 2.4 \\
$55-59$ & 53.4 & 31.4 & 10.0 & 6.81 & 6.3 & 4.2 \\
$60-64$ & 57.0 & 35.5 & 12.0 & 10.24 & 6.5 & 3.8 \\
$65+$ & 62.7 & 32.8 & 12.7 & 7.01 & 4.8 & 1.9 \\
\hline
\end{tabular}

increasing educational level, the proportion of respondents not intending to quit declined, from $64.1 \%$ to $46.3 \%$, and the percentages of respondents who had quit or intended to quit increased. The profile was most favourable for those with college education, but even for this group almost half had never considered giving up their smoking. Of the occupational groups considered, $69.1 \%$ of farmers, $64.5 \%$ of workers, $62.8 \%$ of floating people, and $62.0 \%$ of officers did not intend to give up smoking (fig 2). The medical workers and teachers had slightly higher rates of cessation, but about half did not intend to give up smoking.

About $20 \%$ of ever smokers had stopped smoking at least once, but about half had relapsed. The prevalence rate of reported cessation increased with age (fig 3) above the ages of 25-29 years. The survey questions allowed us to classify the quitters into strata of duration of successful cessation: relapse, $<6$ months, 6-24 months, > 24 months, and unknown duration of quitting. Overall, the corresponding percentages of the former smokers were $20.8 \%, 16.6 \%$, and $37.2 \%$, respectively, and $25.4 \%$ did not give a duration of quitting.

Patterns of cessation varied with educational level (fig 1). The percentage of persons who had ever taken action to quit was highest-about 33\%-for the most educated participants compared with all other groups, for which this percentage was around $20 \%$. Long term quitting was also more common among the most educated $(7.1 \%)$. There was little variation across the four less educated groups. The proportions of relapsed quitters and former smokers were comparable in all of the groups.
Similar analyses were carried out among selected occupational groups (fig 2). Among these occupations, farmers, workers, and floating people had the lowest rates of quitting. Medical workers, teachers, and officers had the highest rates of quitting, including both former smokers and relapsed current smokers, and the highest proportions intending to quit. However, the percentages of former smokers were still low in both groups.

Of participants from urban and rural areas, $11.7 \%$ and $11.4 \%$, respectively, had tried to quit. Relapse rates were quite similar, at $9.0 \%$ (urban) and 9.7\% (rural), respectively.

Respondents who had quit were asked why and allowed to select reasons from a list. Illness was the most common reason overall, selected by $47.3 \%$ of participants who had quit. The second most common reason was illness prevention (34.1\%). Family pressure and economic considerations were given by $15 \%$ and $11 \%$ of responders, respectively. Only a few chose health education and environmental limitation. The reasons for quitting varied with age (table 2). Not surprisingly, the presence of illness was selected more frequently with increasing age. Younger persons more often gave family pressure as the reason for quitting. Costs and the consequences of health education were offered less frequently as the explanation, with restrictions on smoking cited far less frequently. There were also different distributions of reasons for quitting among educational levels, occupations, and income. In general, more smokers with lower educational levels gave up smoking because of illness and cost. For example, among smokers with no schooling, $65 \%$ gave illness as a reason, and $13 \%$ said cost considerations. The same figures among smokers with at least college education level were $37.9 \%$ and $5 \%$, respectively. The trend was the same by income level. Among smokers earning less than 50 RBM per month, $57 \%$ gave illness as a reason, while $19 \%$ said cost. Those same figures among smokers earning more than $1000 \mathrm{RBM}$ per month were $33 \%$ and $0 \%$, respectively. Among farmers, $66 \%$ of quitters listed illness, while $16 \%$ said cost. The same figures among medical workers were $22 \%$ and $0 \%$, respectively, and $40 \%$ chose prevention of disease.

\section{Discussion}

In approaching these survey data, we have viewed smoking cessation not as a dichotomous process but rather as a continuum involving steps that begin with contemplation and culminate with successful cessation. While our questions do not precisely follow those used to establish stages in the stages of change model, the parallel is close and we link to the model as a framework for interpreting the data or reported intent to quit. We found that the majority of China's current smokers $(72 \%)$ are not intending to quit and are presumably in the precontemplation stage. About $20 \%$ have taken action to quit, but half had relapsed. The findings show that China faces an enormous challenge in persuading smokers to stop smoking. Of the approximately 320 million ever 
smokers in China, only 11 million-less than $4 \%$ - have stopped smoking for at least two years, and the majority-205 million-have never contemplated quitting. The number who have relapsed exceeds the number of former smokers who have successfully quit. These findings should figure centrally in formulating smoking cessation programmes, whether at the population or individual levels.

These findings contrast sharply with the high proportions of former smokers in the USA, the UK, and other developed countries. ${ }^{1}$ In fact, in the USA the number of former smokers is now approximately equal to the number of current smokers. ${ }^{13}$ Also in the USA, most smokers report wanting to quit and the majority have made at least one attempt to quit. ${ }^{12}{ }^{13}$ By contrast, only a small proportion of smokers in China have successfully quit and the relapse rate is high (table 1 and fig 3 ). The stages of change model places smokers into five categories with regard to cessation: precontemplation, contemplation, preparation, and action, maintenance or relapse. ${ }^{9}$ While the 1996 survey questions did not have questions to separate contemplation and preparation, the results show that the majority of smokers are not intending to quit (table 1). Recent US data by contrast show that the majority of smokers report being either in the contemplation or preparation stages. ${ }^{14}$ The findings in China indicate that cessation programmes need to focus, at least initially, in moving more smokers to consider quitting - that is, beyond the stage of precontemplation.

Lack of knowledge of the harmful effects of smoking may partially explain the high proportion of participants who do not intend to quit. The 1996 survey showed that knowledge of the many causal associations of smoking with disease was limited. The majority of smokers in the national prevalence survey did not acknowledge the hazard of smoking ${ }^{2} ; 68.6 \%$ of smokers said that the risk of smoking was negligible or small and $8.1 \%$ did not know about any risk. Knowledge of specific diseases caused by smoking was also low, with only a few percent aware that smoking causes heart disease. In the USA and other countries where cessation rates are higher than in China, the majority of smokers acknowledge the health risks. ${ }^{12}{ }^{15}$ Approaches for increasing knowledge among smokers on the health risks of smoking should be developed and tested in China.

The survey also showed that cost did not deter most smokers from smoking. Only about $10 \%$ of smokers identified cost as a reason for quitting, and taxes on cigarettes in China are low in comparison with other countries. ${ }^{16} \mathrm{Par}$ ticipants with lower income, lower educational level, youths, workers, and farmers more often reported cost as a reason for quitting. Price increases appear warranted as a strategy to encourage cessation, even though smokers are already spending around a quarter of their income on cigarettes. One recent analysis suggests that the effect of a price increase in China on smoking might be greater than in developed countries and that there should be a net fiscal gain by the government. ${ }^{16}{ }^{17}$ Experience from other countries shows that smoking by youth is particularly sensitive to price and increased prices would be expected to deter young people in China from smoking. It would be timely for the Chinese government to evaluate its current tobacco taxes and consider raising them. In other countries, increasing prices do reduce smoking somewhat, particularly among youth. ${ }^{18}$ However, the relevance of these data to China is uncertain and research specific to China on this topic is needed.

Studies in other countries have shown the key role that physicians can play in smoking cessation $^{19}$ and strategies have been devised to encourage anti-smoking counselling by physicians. ${ }^{19}$ Smoking by physicians in China contrasts sharply with many western countries where very few physicians now smoke. Medical workers who smoke are a major barrier to cessation efforts because people will not believe health education messages when they see physicians and other health care workers smoking. In China, the smoking patterns of physicians are similar to those of other occupational groups, although in this analysis we found that medical workers have a somewhat more favourable quitting pattern than others (fig 2). It is doubtful that Chinese physicians who smoke offer strong advice about cessation to patients. Researchers need to know more about the messages given to Chinese patients by their physicians on smoking and professional medical societies in China should discourage smoking by their members.

The high relapse rate indicates a need for effective methods for Chinese smokers for maintaining cessation. Some current methods use traditional Chinese medicine including acupuncture and herbs, with many reports on cessation methods in the Chinese literature. ${ }^{20}$ Nicotine replacement therapy and other pharmacologic approaches have not yet been widely used and these methods need specific evaluation in China.

A potential limitation of this survey is its use of self reporting of cigarette smoking without biochemical validation. In other countries, comparison of self report with biochemical markers has shown that some persons say they are former smokers when nicotine levels are consistent with active smoking. ${ }^{12}$ One recent study of southeast Asian immigrants in central Ohio showed misclassification of self reported smoking, with salivary cotinine as the standard. ${ }^{21}$ The tendency to underreport was greater among women. The relevance of these data to our findings is uncertain but replication of a study of this design in China would be useful. Additionally, we have interpreted the survey data within the context of the stages of change model. The appropriateness of this model to smoking in China has not been addressed specifically.

The survey findings suggest that a two pronged national strategy to increase smoking cessation is needed. First, smokers must be mobilised to contemplate quitting and then to take action. Second, effective and evaluated smoking cessation strategies need to be 
developed. Within the framework of a comprehensive model for prevention, many opportunities exist for programmatic action: media campaigns to increase knowledge, increased tobacco taxes, education of health care professionals to strengthen their role in encouraging and sustaining cessation, enhancing support from family and friends, and developing and evaluating smoking cessation methods. Policies now in place leave these opportunities largely unaddressed. The current package warning, "Smoking is harmful for your health," has little impact, according to this survey.

\section{What this paper adds}

China is the world's largest producer and consumer of tobacco, and the number of smokers, especially among younger males, is increasing. Current estimates are that one million people in China will die annually of diseases related to smoking, increasing to two million by around 2025. Some of these deaths can be prevented through cessation, an essential component of tobacco control within a comprehensive intervention strategy.

To plan successful cessation programmes in China, it is necessary to understand the characteristics of Chinese ever smokers. This study uses the 1996 national prevalence survey to examine the distribution of the population of ever smokers with regard to cessation and intent to quit. Results show that most smokers in China do not intend to quit. Successful cessation rates are extremely low, and, unlike in many developed countries, in China the profile of those intending to quit does not differ by educational level or occupation.

\section{Key points}

- $72 \%$ of current smokers in China did not intend to give up smoking in 1996

- About $10 \%$ of ever smokers in China have stopped smoking

- The relapse rate equals the rate of quitting

- Key professional groups (health workers and teachers) have similar cessation patterns to the general population

- The most common reason for quitting was illness

- Smokers in China should be mobilised to contemplate quitting and then to take action
The authors gratefully acknowledge the support of Smith-Kline Beecham and the Rockefeller Foundation.

1 World Health Organization. Tobacco or health: a global status report. Geneva: World Health Organization, 1997.

2 Anon. Smoking and health in China: 1996 national prevalence survey of smoking pattern. In: Yang $\mathrm{G}$, Lixin $\mathrm{F}$, Zhengiing $\mathrm{H}$, et al, eds. Beijing: China Science and Technology Press, 1996.

3 Yang G, Lixin F, Tan J, et al. Smoking in China: findings of the 1996 national prevalence survey. $7 A M A$ 1999; 282:1247-53.

4 Niu S-R, Yang G-H, Chen Z-M, et al. Emerging tobacco hazards in China: 2. Early mortality results from a prospective study. BMF 1998;317:1423-4.

5 Liu B-Q, Peto R, Chen Z-M, et al. Emerging tobacco hazards in China: 1 . Retrospective proportional mortality study of one million deaths. BMF 1998;317:1411-22.

6 WHO. The World Health Organization report 1997. Conquering suffering; enriching humanity. Geneva: WHO, 1997.

7 Peto R, Lopez AD, Boreham J, et al. Mortality from smoking worldwide. Br Med Bull 1996;52:12-21.

8 Institute for the Study of Smoking Behavior and Policy. The policy implications of the 1986 Surgeon General's report on for the Study of Smoking Behavior and Policy, 1988.

9 Prochaska JO, DiClemente CC. Stages and processes of self-change of smoking: toward an integrative model of change. F Consult Clin Psychol 1983;51:390.

10 Yang G-H, Zhen X, Zhen G. Selection of DSP points in the second stage and their presentation. $\mathcal{f}$ Chinese Epidemiol 1992;13:79-81.

11 World Health Organization. Guidelines for the conduct of tobacco smoking surveys for the general population. Technica Document No. WHO/SMO/83.4. Geneva: WHO, 1983.

12 US Department of Health and Human Services. The health benefits of smoking cessation. A report of the Surgeon General, 1990. Rockville, Maryland: Public Health Service, Centers for Disease Control, Office on Smoking and Health, 1990. (DHHS Publication No (CDC) 90-8416.)

13 US Department of Health and Human Services. Preventing tobacco use among young people. A report of the Surgeon General, 1994. Atlanta, Georgia: Public Health Service, Centers for Disease Control and Prevention, Office on Smoking and Health, 1994. (US Government Printing Office Publication No S/N 017-001-00491-0.)

14 Velicer WF, Fava JL, Prochaska JO, et al. Distribution of smokers by stage in three representative samples. Prev Med 1995;24:401-11.

15 Brownson RC, Jackson-Thompson J, Wilkerson JC, et al. Demographic and socioeconomic differences in beliefs about the health effects of smoking. Am f Public Health 1992;82:99-103.

16 Townsend J. The role of taxation policy in tobacco control. In: Abedian I, van der Merwe R, Wilkins N, Jha P, eds. The economics of tobacco control. Towards an optimal policy mix. Mowbray, W Cape: Edson-Clyde Press, 1998;85-101.

$17 \mathrm{Hu}$ T-W, Xu X-P, Keeler T. Earmarked tobacco taxes: lessons learned. In: Abedian I, van der Merwe R, Wilkins $\mathrm{N}$, Jha P, eds. The economics of tobacco control. Towards an optimal policy mix. Mowbray, W Cape: Edson-Clyde Press, optimal policy

18 The World Bank. Curbing the epidemic: governments and the economics of tobacco control. Washington DC: The International Bank for Reconstruction and Development, 1999.

19 Fiore MC, Bailey WC, Cohen SJ, et al. Smoking cessation. Clinical Practice Guideline No 18. Rockville, Maryland: US Department of Health and Human Services, Public Health Service, Agency for Health Care Policy and Research, April 1996. (AHCPR Publication No 960692.); <http://text.nlm.nih.gov>.

20 Li X, Fang X, Stanton B. Cigarette smoking among Chinese adolescents and its association with demographic characteristics, social activities, and problem behaviors. Substance Use and Misuse 1996;31:545-63.

21 Wewers ME, Dhatt RK, Moeschberger ML, et al. Misclassification of smoking status among Southeast Asian adult immigrants. Am f Resp Crit Care Med 1995; 152(6Pt1):1917-21. 